\title{
Custos diretos da dor lombar em hospitais financiados pelo Sistema Único de Saúde
}

\author{
Direct costs of low back pain in hospitals \\ financed by the Unified Health System
}

\section{Alysson Geraldo Mendonça' ${ }^{1}$ (1) Vinicius Cunha Oliveira ${ }^{2}$ (1) Letícia Soares Fonseca ${ }^{3}$ (1) Murilo Xavier Oliveira ${ }^{4}$ (1)}

${ }^{1}$ Autor para correspondência. Universidade do Estado de Minas Gerais (Belo Horizonte). Minas Gerais, Brasil. alysson_mendonca@hotmail.com 2-4Universidade Federal dos Vales do Jequitinhonha e Mucuri (Diamantina). Minas Gerais, Brasil. vcunhaoliveira@gmail.com, leticia.soares97@outlook.com, murilo.xavier@ufvjm.edu.br

RESUMO | INTRODUÇÃO: A dor lombar é um sintoma altamente incapacitante, que leva a um impacto social negativo para as pessoas que experimentam o sintoma, e econômico para os cofres públicos de diversos países, com gastos excessivos e por vezes desnecessários com procedimentos não resolutivos. OBJETIVOS: Relatar o número de procedimentos clínicos e cirúrgicos e os custos diretos da dor lombar em hospitais financiados pelo Sistema Único de Saúde. MÉTODOS: Os dados sobre os procedimentos clínicos e cirúrgicos foram coletados no Sistema de Informações Hospitalares no website do DATASUS, no período entre 2013 e 2018. Realizou-se análise descritiva dos dados. RESULTADOS: nos seis anos analisados os procedimentos para tratamento da dor lombar custaram financeiramente $R \$ 24.427 .238$. Estes custos aumentaram de forma expressiva ao longo dos anos observados. Realizou-se 1.689 procedimentos cirúrgicos e cada um custou em média $\mathrm{R} \$$ 3.290. A região sudeste do país foi a que teve maior gasto financeiro para o tratamento do sintoma ( $R \$ 12.442 .930$ ). CONCLUSÃO: O custo da dor lombar ao longo dos anos para o sistema público está aumentando rapidamente e provavelmente é impulsionado pelo aumento no número de cirurgias.

PALAVRAS-CHAVE: Custos diretos. Dor lombar. Hospitais. Brasil.

\begin{abstract}
INTRODUCTION: Low back pain is a highly disabling symptom, which leads to a negative social impact for people who experience the symptom, and economic, for public coffers in several countries, with excessive and sometimes unnecessary expenses with non-resolving procedures. OBJECTIVES: To report the number of clinical and surgical procedures and the direct costs of low back pain in hospitals financed by the Unified Health System. METHODS: The data on clinical and surgical procedures were collected in the Hospital Information System on the DATASUS website between 2013 and 2018. A descriptive analysis of the data was carried. RESULTS: In the six years analyzed, the procedures for the treatment of low back pain cost $R \$ 24,427,238$. These costs have increased significantly over the years observed. 1,689 surgical procedures were performed, and each cost an average of $R \$ 3,290$. The southeastern region of the country was the one that had the greatest financial expenditure for the treatment of the symptom ( $R \$ 12,442,930)$. CONCLUSION: The cost of low back pain over the years to the public system is increasing rapidly and is probably driven by the increase in the number of surgeries.
\end{abstract}

KEYWORDS: Direct costs. Low back pain. Hospitals. Brazil. 


\section{Introdução}

A dor lombar (DL) atualmente é a principal causa de incapacidade física em todo o mundo ${ }^{1}$, e isso resulta em altos custos para os sistemas de saúde e para as pessoas que experimentam o sintoma ${ }^{2}$. Esses custos podem ser tanto diretos (atendimentos médicos, fisioterapêuticos) quanto indiretos (dias perdidos de trabalho e perda de produtividade) ${ }^{3}$. Estudos recentes têm buscado estabelecer o custo total da DL e comparar os resultados obtidos entre vários países. Entretanto, ocorre variação nessas estimativas, devido a diferentes métodos operacionais e de coleta de dados nos sistemas de saúde em diferentes países".

O que é descrito na literatura atual é que o número de casos de DL aumentou nos países de alta renda, e assim é provável que o impacto econômico do sintoma também esteja aumentando nestes países ${ }^{5-7}$. $\mathrm{Na}$ Suécia, o custo total direto e indireto estimado da DL foi de 740 milhões de euros em 2011, o que correspondeu a 78 euros por pessoa no país no ano ${ }^{3}$. Nos Estados Unidos da América, os custos médicos da DL aumentaram de US $\$ 4695$ por pessoa, em 1997, para US \$ 6096, em 2015². Na Alemanha, os custos totais da DL por paciente foram estimados em 1322 euros por $a n o^{8}$.

Já nos países de média e baixa renda, faltam dados conclusivos. No Brasil, por exemplo, poucos estudos epidemiológicos buscaram descrever as características ou os custos financeiros da DLํ․ Desta forma, os dados sobre o uso de recursos no gerenciamento da DL nos três níveis de assistência à saúde são inconclusivos. Até onde sabemos, há informações muito limitadas na literatura sobre os custos diretos da DL, e se esses custos estão aumentando ou diminuindo ao longo do tempo.

Este estudo tem como objetivo analisar e apresentar dados das notificações de DL em hospitais financiados pelo Sistema Único de Saúde (SUS), descrevendo a frequência de procedimentos clínicos e cirúrgicos realizados, bem como os custos financeiros desses procedimentos de 2013 a 2018.

\section{Desenho do estudo e coleta dos dados}

Estudo epidemiológico longitudinal ecológico do tipo painel, com informações públicas disponíveis no website do DATASUS (Departamento de Informática do Sistema Público de Saúde). Foi realizado download de dados no período entre 2013 e 2018 no Sistema de Informações Hospitalares (SIH), que possui as informações sobre os atendimentos hospitalares que foram financiadas pelo SUS ${ }^{10}$. Os métodos seguiram as diretrizes da Declaração Fortalecendo a Comunicação de Estudos Observacionais em Epidemiologia (STROBE): Diretrizes para a Comunicação de Estudos Observacionais ${ }^{11}$.

\section{Extração e codificação dos dados de acordo com a Classificação Internacional de Doenças (CID-10)}

Após o download das informações hospitalares no site do DATASUS, os dados foram transferidos para o software TABWIN 4.1.3. O TABWIN 4.1.3, que é fornecido pelo Ministério da Saúde do Brasil e permite a análise exploratória dos dados de hospitais públicos, fornecendo características epidemiológicas e sociodemográficas das condições de saúde no país e nas macrorregiões.

Para a análise exploratória dos dados no TABWIN 4.1.3, foi realizada a codificação pela CID-10. A CID10 padroniza as doenças e os problemas relacionados à saúde, usando a Nomenclatura Internacional de Doenças da Organização Mundial da Saúde (OMS) como referência. Os seguintes códigos da CID-10, capítulo XIII [Doenças do sistema musculoesquelético e do tecido conjuntivo (M00-M99] foram utilizados: M54.4 (lombalgia com ciática), M54.5 (dor lombar baixa), M54.8 (outras dorsalgias) e M54.9 (dorsalgias não especificadas). Utilizamos estratégia de codificação parecida com estudos anteriores para designar o sintoma "dor lombar", como no estudo de Jöud et al. ${ }^{12}$, uma vez que nesse estudo esses códigos foram escolhidos através de consenso entre especialistas da área, como os mais relevantes para DL. 
Para cada código da CID-10 utilizado é considerada uma notificação hospitalar para DL, e um procedimento hospitalar realizado é registrado. No caso do presente estudo, para cada código da CID-10 utilizado no momento da conclusão da Autorização de Internação Hospitalar (AlH), que é o documento que alimenta o banco de dados do DATASUS, é registrado um procedimento clínico ou cirúrgico para o paciente notificado.

\section{Variáveis de interesse}

Os seguintes dados foram extraídos de 2013 a 2018:

- Frequência e custos diretos dos procedimentos clínicos (procedimentos não invasivos). Inclui o custo de todos os procedimentos realizados desde o momento da internação até a alta hospitalar, como: materiais hospitalares, medicamentos utilizados e serviços auxiliares de diagnóstico e terapia, exames de imagem, procedimentos ortopédicos não invasivos e tratamento de complicações clínicas.

- Frequência e custos diretos dos procedimentos cirúrgicos (pacientes que receberam alguma intervenção manual ou instrumental invasiva no corpo para diagnosticar, tratar ou curar). Inclui os custos de todos os procedimentos realizados antes e após a cirurgia do paciente, como biópsia, artrodese, discotomias, ressecções de elementos vertebrais, tratamentos pós-cirúrgicos e tratamento de complicações cirúrgicas, materiais hospitalares, anestesia e medicamentos relacionados ao tratamento de cirurgias para DL $\frac{13}{}$.

\section{Análise dos dados}

Foi realizada análise descritiva dos dados, e estes foram apresentados em uma tabela contendo o número total e os custos diretos dos procedimentos clínicos e cirúrgicos, e o valor médio de cada procedimento realizado. Os gráficos representam o número total e os custos diretos dos procedimentos nos seis anos nas cinco regiões brasileiras e no país (cálculos mostrados abaixo). Os custos financeiros foram apresentados de duas maneiras, na moeda brasileira ( $R \$$ ) e também convertidos em dólares norte-americanos (US \$), pela taxa de câmbio de 29 de agosto de 2019: US \$1 = R \$4,172 (moeda brasileira). Os gráficos e tabelas foram apresentados em US \$.

Custo do procedimento no ano $=$

Custo total de procedimentos clínicos ou cirúrgicos no ano para dor lombar Número total de procedimentos clínicos ou cirurgicos no ano para dor lombar

A análise dos dados foi realizada na versão SPSS 22.0 (SPSS Inc., Chicago, USA).

\section{Aspectos éticos}

O estudo foi registrado no Comitê de Ética e Pesquisa da Universidade Federal dos Vales do Jequitinhonha e Mucuri (número do processo: 99571018.8.0000.5108), e foi liberado para execução, uma vez que se trata de estudo com dados públicos. 


\section{Resultados}

\section{Número de procedimentos clínicos e cirúrgicos}

Nos seis anos, foram notificados 59.954 casos de DL no $\mathrm{SIH}$, sendo 1.689 procedimentos cirúrgicos e 58.265 procedimentos clínicos. Ocorreu aumento no número de cirurgias para casos de DL ao longo dos seis anos, passando de duas cirurgias notificadas em 2013 para 642 em 2018 (Figura 1). Em relação aos procedimentos clínicos, estes se mantiveram estáveis ao longo dos seis anos, com um pequeno acréscimo quando comparado 2013 com 2018. (Tabela 1).

\section{Custo direto dos procedimentos clínicos e cirúrgicos}

Nos seis anos analisados, os custos financeiros da DL para o sistema público de saúde brasileiro foram de $R \$ 24.427 .238$ (US \$ 5.857.851), com um aumento nos custos diretos das notificações de $\mathrm{R} \$ 2.795 .851$ (US \$ 670.468), em 2013, para R\$ 4.907.198 (US \$ 1.176.786), em 2018 (aumento de $R \$ 2.111 .346 /$ US $\$$ 506.318).

Os custos diretos totais dos procedimentos clínicos e cirúrgicos aumentaram ao longo dos anos. Os procedimentos clínicos aumentaram de R\$2.793.854 (US \$ 669.989) em 2013 para R\$3.065.926 (US \$ 735.234), em 2018, e os procedimentos cirúrgicos aumentaram de $R \$ 1.997$ (US \$ 479), em 2013, para $R \$ 1.841272$ (US \$ 441.552), em 2018 (Figura 2). O custo direto médio no período foi de $\mathrm{R} \$ 321$ (US \$77) para cada procedimento clínico e R $\$ 3.290$ (US \$ 789) para cada cirurgia realizada.

Número de procedimentos nas regiões brasileiras Entre as cinco regiões do Brasil, a região sudeste foi a que mais realizou procedimentos nos seis anos $(n=30.055)$, sendo 1095 cirurgias e 28.960 procedimentos clínicos. A região norte do país apresentou o menor número de procedimentos nos seis anos ( $\mathrm{n}=$ 4.567), dos quais 4.558 foram procedimentos clínicos e 9 cirúrgicos (Figura 3).

\section{Custos diretos dos procedimentos clínicos e cirúrgicos nas regiões brasileiras}

A região sudeste brasileira foi a que gastou mais recursos financeiros no período para tratamento da DL em hospitais do SUS R\$12.442.930 (US \$ 2.979.120). $E$ a região norte gastou menos recursos $R \$ 1.087 .144$ (US \$ 260.706) (Figura 4). A região centro-oeste gastou mais em cada cirurgia (média: R\$5.571 - US \$ 1.336 / cirurgia), enquanto a região nordeste gastou menos (média: R\$2.214 - US \$531 / cirurgia). Em relação aos procedimentos clínicos, a região sul gastou mais (média: $R \$ 388$ - US \$93 / procedimento clínico) e a região norte gastou menos recursos financeiros (média: $R \$$ 229 - US \$ 55 / procedimento clínico).

Figura 1. Número total de cirurgias notificadas para os casos de dor lombar ao longo dos anos em hospitais financiados pelo SUS

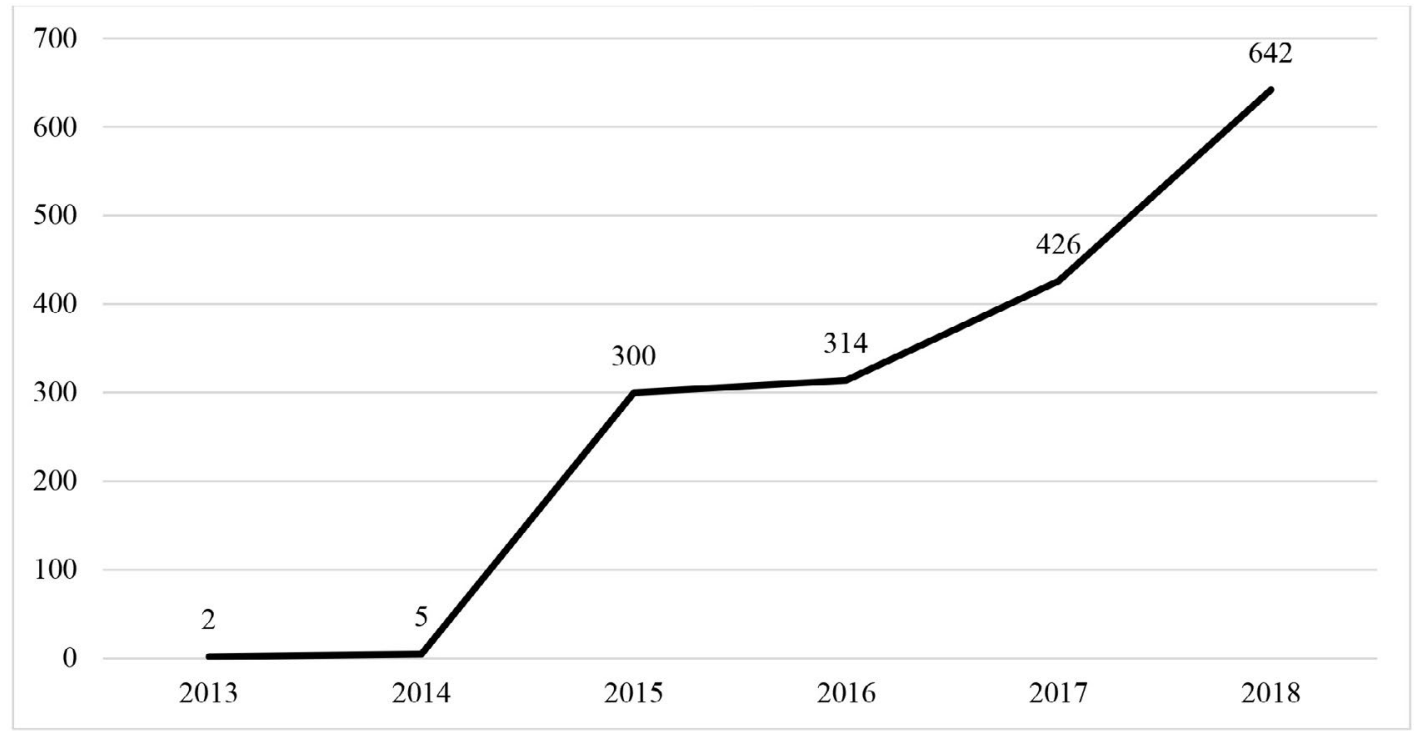


Tabela 1. Número total de procedimentos clínicos e cirúrgicos notificados; custos diretos de procedimentos clínicos e cirúrgicos; e custo direto médio com cada procedimento clínico e cirúrgico realizado entre 2013 e 2018 para notificações de dor lombar em hospitais financiados pelo SUS no Brasil

\begin{tabular}{|c|c|c|c|c|c|c|}
\hline & 2013 & 2014 & 2015 & 2016 & 2017 & 2018 \\
\hline $\begin{array}{l}\text { Total de procedimentos } \\
\text { cirúrgicos por ano }\end{array}$ & 2 & 5 & 300 & 314 & 426 & 642 \\
\hline $\begin{array}{l}\text { Total de procedimentos } \\
\text { clínicos por ano }\end{array}$ & 9.358 & 10.122 & 9.867 & 9.634 & 9.699 & 9.585 \\
\hline $\begin{array}{r}\text { Total de } \\
\text { procedimentos }\end{array}$ & 9.360 & 10.127 & 10.167 & 9.948 & 10.125 & 10.227 \\
\hline $\begin{array}{l}\text { Custos diretos dos } \\
\text { procedimentos cirúrgicos }\end{array}$ & $\mathrm{R} \$ 1.997$ & $\mathrm{R} \$ 12.009$ & $\mathrm{R} \$ 953.704$ & $\mathrm{R} \$ 1.169 .926$ & $R \$ 1.620 .557$ & $\mathrm{R} \$ 1.841 .271$ \\
\hline $\begin{array}{l}\text { Custos diretos dos } \\
\text { procedimentos clínicos }\end{array}$ & $\mathrm{R} \$ 2.793 .854$ & $\mathrm{R} \$ 3.393 .808$ & $\mathrm{R} \$ 3.323 .615$ & $\mathrm{R} \$ 3.185 .909$ & $\mathrm{R} \$ 3.064 .633$ & $\mathrm{R} \$ 3.065 .925$ \\
\hline Custos diretos totais & $\mathrm{R} \$ 2.795 .851$ & $\mathrm{R} \$ 3.405 .822$ & $\mathrm{R} \$ 4.277 .323$ & $\mathrm{R} \$ 4.355 .840$ & $\mathrm{R} \$ 4.685 .195$ & $\mathrm{R} \$ 4.907 .197$ \\
\hline $\begin{array}{l}\text { Custo direto médio de } \\
\text { cada cirurgia realizada }\end{array}$ & $\mathrm{R} \$ 999,29$ & $\mathrm{R} \$ 2.402$ & $\mathrm{R} \$ 3.179$ & $\mathrm{R} \$ 3.725$ & $\mathrm{R} \$ 3.804$ & $\mathrm{R} \$ 2.868$ \\
\hline $\begin{array}{l}\text { Custo direto médio de } \\
\text { cada procedimento } \\
\text { clínico }\end{array}$ & $\mathrm{R} \$ 298,53$ & $\mathrm{R} \$ 335,30$ & $\mathrm{R} \$ 336,85$ & $\mathrm{R} \$ 330,68$ & $\mathrm{R} \$ 315,96$ & $\mathrm{R} \$ 319,88$ \\
\hline
\end{tabular}

Figura 2. Custo direto total, custo direto com procedimentos clínicos e cirúrgicos ao longo dos anos para as notificações de dor lombar em hospitais financiados pelo SUS

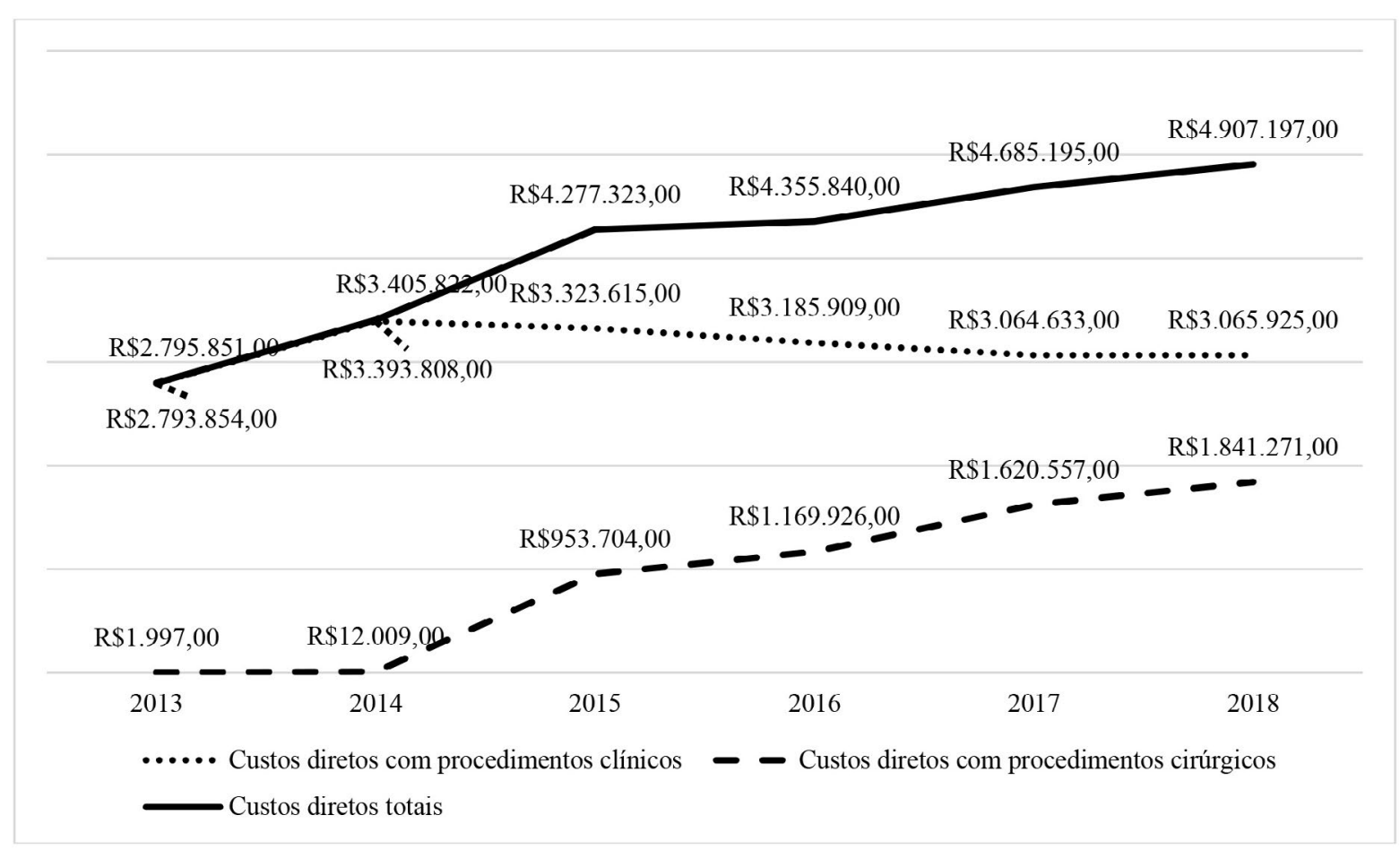




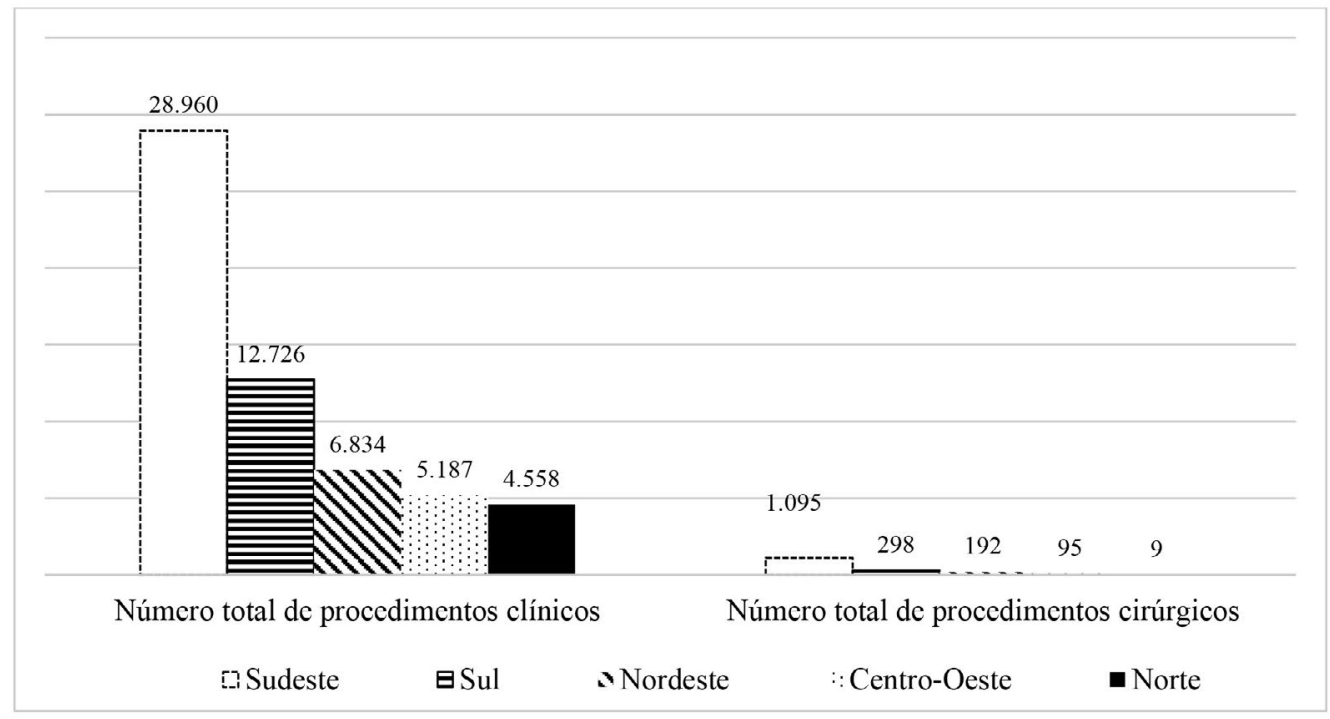

Figura 4. Custo total dos procedimentos em cada região ao longo dos anos analisados

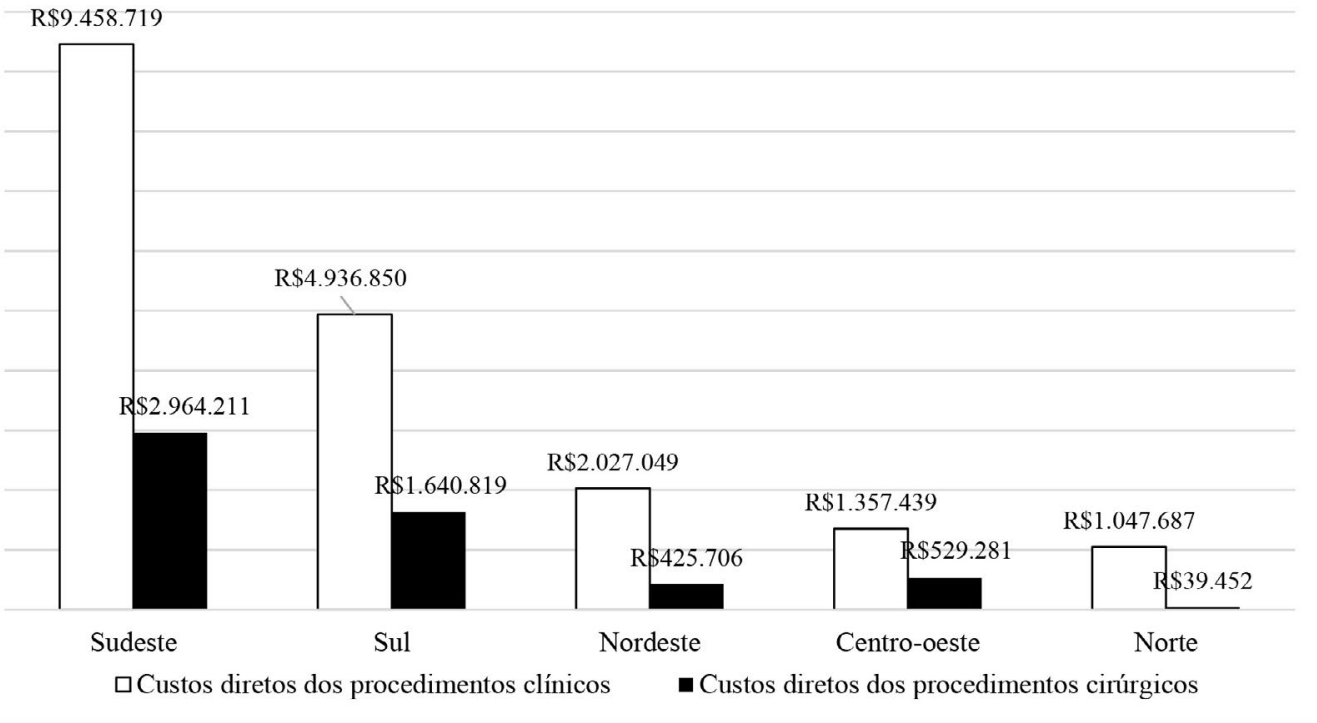




\section{Discussão}

Embora o número de notificações de DL em hospitais públicos brasileiros tenha se mantido praticamente estável entre 2013 e 2018, houve aumento substancial no número de cirurgias para o tratamento do sintoma. Além disso, os custos diretos para o tratamento da DL quase dobraram entre o período, com o maior número de notificações e o maior custo direto na região sudeste do país.

A eficácia dos procedimentos cirúrgicos para casos de DL tem sido questionada na literatura recente. Em muitos casos, a intervenção cirúrgica é baseada em exames de imagem, o que resulta em procedimentos e gastos desnecessários, maior número de dias perdidos no trabalho, e baixo percentual de resolução dos sintomas, o que está associado a reoperações ${ }^{221}$. Os resultados do presente estudo demonstram um grande aumento no número e nos custos financeiros das cirurgias ao longo dos anos analisados, o que está em desacordo com as evidências atuais para o tratamento do sintoma. Esses achados são de extrema importância para o sistema público de saúde brasileiro, e para a implementação de novas políticas voltadas para mudanças no tratamento da DL em centros hospitalares, a fim de evitar cirurgias desnecessárias e seus altos custos, seguindo as recomendações clínicas atuais ${ }^{16,17}$.

Por exemplo, programas de "segunda opinião" para cirurgia na coluna vertebral podem desempenhar um papel importante na redução de tratamentos desnecessários ${ }^{18,19}$. O objetivo desses programas é obter uma segunda opinião médica sobre pacientes com indicação de cirurgia primária ${ }^{18,20}$. Em um estudo envolvendo 166 pacientes com indicações para cirurgia na coluna vertebral no Centro de Referência de Tratamento da Coluna do Hospital Israelita Albert Einstein, no Brasil, 112 pacientes não foram submetidos a cirurgia após receber uma segunda avaliação e receberam tratamento conservador, levando a uma economia de $158,6 \%$ como resultado de um programa da "segunda opinião"1․

Ainda sobre o número de cirurgias, é importante ressaltar que a partir de 2015 ocorreu um aumento muito significativo no número de cirurgias para DL. Este aumento pode estar condicionado a falta de codificação adequada antes de 2015, com a CID-10 incorreta.
Esse achado é importante para médicos e profissionais que trabalham diretamente com o sintoma, para que no futuro possam formular e ter um consenso sobre a codificação mais adequada para casos de DL, não só em hospitais públicos, mas em toda rede de assistência à saúde brasileira.

Em relação às regiões brasileiras, a região sudeste do país realizou mais procedimentos clínicos e cirúrgicos e, consequentemente, gastou mais recursos financeiros para o tratamento da DL. Em um estudo sobre doenças da coluna vertebral no Brasil, com dados de 2016, Carregaro et al. $\underline{21}$, também relatam que as regiões sudeste e sul gastaram mais recursos financeiros para o tratamento dessas condições. Além disso, a região sudeste é a mais populosa do país $\frac{22}{}$, possuindo provavelmente o maior número de profissionais de saúde e serviços hospitalares, o que pode resultar em um número maior e mais eficiente de registros no sistema que alimenta o banco de dados do DATASUS. No entanto, são necessários mais estudos observacionais de base populacional para determinar o motivo destas diferenças nos custos entre as regiões brasileiras.

A comparação com estudos de custo-doença entre países é complicada, uma vez que existem diferenças entre os sistemas de saúde dos países $8,23,24$. Uma revisão sistemática com estudos de países de alta renda que abordam os custos diretos, indiretos e totais da DL relata que, apesar das diferenças metodológicas entre os estudos analisados, os custos econômicos para o tratamento da DL representam um ônus substancial para a sociedade ${ }^{25}$. Em Portugal, os custos indiretos direcionados para a pessoas incapacitadas pela DL no ano de 2010 foi de 458,91 milhões de euros ${ }^{23}$. $\mathrm{Na}$ Holanda, os custos diretos foram de $€ 3,5$ bilhões em 2007, apesar das mudanças na legislação que visam reduzir os custos relacionados a essa condição ${ }^{24}$.

Os achados deste estudo mostram custos diretos mais baixos quando comparados a alguns países de alta renda $3,8,23,24$. No entanto, essa diferença nos custos financeiros pode ser explicada pelo fato de ter sido utilizado no presente estudo apenas dados de um serviço público de saúde no Brasil (hospitais). Assim, esses valores provavelmente seriam maiores se considerássemos outros níveis de assistência do sistema de saúde brasileiro, bem como o setor privado, e os custos indiretos relacionados à condição. 
Carregaro et al..21, em seu estudo sobre os custos de distúrbios da coluna vertebral no Brasil em 2016, relataram resultados diferentes dos apresentados neste estudo, com um custo de US $\$ 71,4$ milhões naquele ano. Essa diferença de valores é justificável, pois o estudo considerou todos os distúrbios da coluna vertebral e, no presente estudo, apenas quatro códigos da CID-10 foram utilizados para representar o sintoma da DL. Além disso, Carregaro et al. 21, incluíram dados de outros serviços de assistência à saúde, enquanto o presente estudo utilizou apenas dados da rede hospitalar pública brasileira. No entanto, os resultados de ambos os estudos indicam uma carga financeira substancial para o tratamento e gerenciamento da DL no sistema de saúde brasileiro, e sugerem que pesquisas adicionais possam identificar as principais causas dessa carga, bem como áreas em que a carga pode ser reduzida.

\section{Limitação}

O banco de dados utilizado (SIH/SUS) é alimentado com informações inseridas por diferentes profissionais de saúde que trabalham no sistema hospitalar brasileiro. Assim, alguns casos podem ter sido subnotificados ou preenchidos incorretamente, com outros códigos da CID-10, além destes que foram utilizados no presente estudo. No banco de dados não há informações exatas sobre a característica da dor (aguda, subaguda ou crônica). Isso limita o entendimento sobre a associação do tipo de dor versus a destinação de recursos para o tratamento do sintoma. Por fim, é importante ser considerado que utilizamos apenas quatro códigos CID-10 para designar o sintoma "dor lombar". Sabendo que profissionais de saúde codificam o sintoma de maneiras distintas, não possuindo uma padronização universal para essa codificação, é plausível considerar a perda de algumas notificações. No entanto, apesar das possíveis limitações listadas, é necessário considerarmos que o banco de dados do SIH/SUS é confiável e é atualmente a melhor maneira para termos conhecimentos epidemiológicos da saúde da população brasileira.

\section{Conclusão}

Os procedimentos cirúrgicos para DL quase dobraram no período de seis anos, e, consequentemente, os custos diretos para tratamento do sintoma aumentaram consideravelmente no serviço hospitalar público brasileiro. Os resultados aqui apresentados são importantes para futuras discussões sobre a meIhor forma de gerenciamento e tratamento da $\mathrm{DL}$, afim de minimizar estes custos e procedimentos.

\section{Contribuições dos autores}

Mendonça AG participou da concepção e delineamento do projeto de pesquisa, coleta e análise estatística dos dados, interpretação dos resultados e redação do artigo científico. Oliveira, VC e Oliveira, MX participaram da concepção e delineamento do projeto de pesquisa, confecção e revisão do artigo científico. Fonseca, LS participou da coleta e análise dos dados.

\section{Conflitos de interesses}

Nenhum conflito financeiro, legal ou político envolvendo terceiros (governo, empresas e fundações privadas, etc.) foi declarado para nenhum aspecto do trabalho submetido (incluindo, mas não se limitando a subvenções e financiamentos, participação em conselho consultivo, desenho de estudo, preparação de manuscrito, análise estatística, etc.).

\section{Referências}

\begin{abstract}
1. Hartvigsen J, Hancock MJ, Kongsted A, Louw Q, Ferreira ML, Genevay S, et al. What low back pain is and why we need to pay attention. Lancet. 2018;391(10137):2356-67. https://doi. org/10.1016/s0140-6736(18)30480-x
\end{abstract}

2. Martin BI, Deyo RA, Turner JA, Comstock BA, Hollingworth W, Sullivan SD. Expenditures and Health Status Among Adults With Back and Neck Problems. JAMA. 2015;299(6):656-64. https://doi. org/10.1001/jama.299.6.656

3. Olafsson G, Jonsson E, Fritzell P, Hägg O, Borgström F. Cost of low back pain : results from a national register study in Sweden. Eur Spine J. 2018;27(11):2875-81. https://doi.org/10.1007/s00586$\underline{018-5742-6}$ 
4. Vos T, Abajobir AA, Abate KH, Abbafati C, Abbas KM, Abd-Allah $F$, et al. Global, regional, and national incidence, prevalence, and years lived with disability for 328 diseases and injuries for 195 countries, 1990-2016: A systematic analysis for the Global Burden of Disease Study 2016. Lancet. 2017;390(10100):1211-59. https:// doi.org/10.1016/S0140-6736(17)32154-2

5. Slade SC, Kent P, Patel S, Bucknall T, Buchbinder R. Barriers to primary care clinician adherence to clinical guidelines for the management of low back pain : protocol of a systematic review and meta-synthesis of qualitative studies. Clin J Pain. 2016;32(9):800-16. https://doi.org/10.1097/ ajp.0000000000000324

6. Buchbinder R, Tulder M, Öberg B, Costa LM, Woolf A, Schoene $M$, et al. Low back pain: a call for action. Lancet. 2018;391(10137):2384-8. https://doi.org/10.1016/S01406736(18)30488-4

7. Foster NE, Anema JR, Cherkin D, Chou R, Cohen SP, Gross DP, et al. Prevention and treatment of low back pain: evidence, challenges, and promising directions. Lancet. 2018;391(10137):2368-83. https://doi.org/10.1016/s01406736(18)30489-6

8. Wenig CM, Schmidt CO, KohImann T, Schweikert B. Costs of back pain in Germany. Eur J Pain. 2009;13(3):280-6. https://doi. org/10.1016/j.ejpain.2008.04.005

9. Nascimento PRC, Costa LOP. Prevalência da dor lombar no Brasil: uma revisão sistemática. Cad. Saúde Pública [online]. 2015;31(6):1141-55. https://doi.org/10.1590/0102-311X00046114

10. Lessa FJD, Mendes ACG, Farias SF, Sá DA, Duarte PO, Melo Filho DA. Novas Metodologias para Vigilância Epidemiológica : Uso do Sistema de Informações Hospitalares - SIH / SUS. Inf Epidemiológico do SUS. 2000;9(supl.1):3-27. https://doi. org/10.5123/S0104-16732000000500001

11. Elm E, Altman DG, Egger M, Pocock SJ, Gøtzsche PC, Vandenbroucke JP. Annals of Internal Medicine Academia and Clinic The Strengthening the Reporting of Observational Studies in Epidemiology ( STROBE ) Statement : Guidelines for Reporting. J Clin Epidemiol. 2007;147(8):573-8. https://doi.org/10.7326/00034819-147-8-200710160-00010

12. Jöud A, Petersson IF, Englund M. Low back pain: Epidemiology of consultations. Arthritis Care Res. 2012;64(7):1084-8. Citado em: PMID: 22337573

13. Ministério da Saúde (Brasil), Secretaria de Atenção à Saúde, Departamento de Regulação, Avaliação e Controle/Coordenação Geral de Sistemas de Informação. Manual técnico operacial do sistema. Brasília: Ministério da Saúde; 2017. Disponível em: http:// www.saude.sp.gov.br/resources/ses/perfil/gestor/homepage/ auditoria/manuais/manual_sih_janeiro_2017.pdf

14. Martin BI, Mirza ÃSK, Spina N, Spiker ÃWR, Lawrence B, Brodke DS. Trends in Lumbar Fusion Procedure Rates and Diseases in the United States, 2004 to 2015. Spine J. 2019;44(5):369-76. https:// doi.org/10.1097/BRS.0000000000002822
15. Martin BI, Mirza SK, Comstock BA, Gray DT, Kreuter W, Deyo RA. Reoperation Rates Following Lumbar Spine Surgery and the Influence of Spinal Fusion Procedures. Spine J. 2007;32(3):382-7. https://doi.org/10.1097/01.brs.0000254104.55716.46

16. Österman H, Sund R, Seitsalo S, Keskimaki I. Risk of Multiple Reoperations After Lumbar Discectomy. Spine J. 2003;28(6):621-7. https://doi.org/10.1097/01.brs.0000049908.15854.ed

17. Lemmers GPG, Lankveld W, Westert GP, Wees PJ, Staal JB. Imaging versus no imaging for low back pain : a systematic review, measuring costs, healthcare utilization and absence from work. Eur Spine J. 2019;28(5):937-50. https://doi.org/10.1007/s00586019-05918-1

18. Lenza M, Buchbinder R, Staples MP, Santos OFP, Brandt RA, Lottenberg $C L$, et al. Second opinion for degenerative spinal conditions : an option or a necessity ? A prospective observational study. BMC Musculoskelet Disord. 2017;354:1-12. https://dx.doi. org/10.1186\%2Fs12891-017-1712-0

19. Viola DCM, Lenza M, Almeida SLF, Santos OFP, Cendoroglo Neto $M$, Lottenberg CL, et al. Redução do custo em cirurgia de coluna em um centro especializado de tratamento. Einstein (São Paulo). 2013;11(1):102-7. http://dx.doi.org/10.1590/S167945082013000100018

20. Hooff ML, Jacobs WC, Willems PC, Wouters MW, Kleuver $\mathrm{M}$, Peul WC, et al. Evidence and practice in spine registries $A$ systematic review, and recommendations for future design of registries. Acta Orthop. 2015;86(5):534-44. https://dx. doi. org/10.3109\%2F17453674.2015.1043174

21. Carregaro RL, Silva EN, Tulder M. Direct healthcare costs of spinal disorders in Brazil. Int J Public Health. 2018;64:965-74. https://doi.org/10.1007/s00038-019-01211-6

22. Instituto de pesquisa econômica aplicada (Brasil). Desenvolvimento Humano Nas Macrorregiões Brasileiras. Brasília: PNUD: IPEA: FJP; 2016. Disponível em: http://www.ipea.gov.br/ portal/images/stories/PDFs/livros/livros/20160331_livro-idhm.pdf

23. Gouveia M, Augusto M. Custos indirectos da dor crónica em Portugal. Rev Port Saúde Pública. 2011;29(2):100-7. https://doi. org/10.1016/S0870-9025(11)70013-X

24. Lambeek LC, Tulder MW, Swinkels ICS, Koppes LLJ, Anema JR, Mechelen W. The Trend in Total Cost of Back Pain in the Netherlands in the Period 2002 to 2007. Spine. 2011;36(13):10508. https://dx.doi.org/10.1097/BRS.0b013e3181e70488

25. Edwards J, Hayden J, Asbridge M, Gregoire B, Magee K. Prevalence of low back pain in emergency settings: a systematic review and meta-analysis. BMC Musculoskelet Disord. 2017;18(1):143. https://dx.doi.org/10.1186\%2Fs12891-017-1511-7 